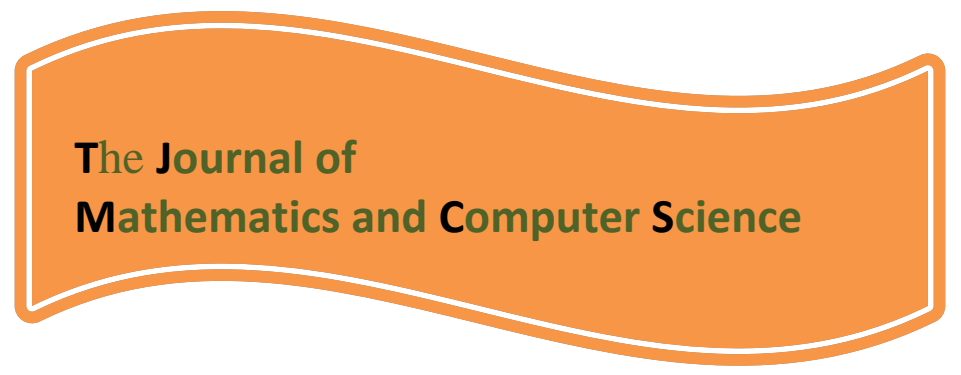

Available online at

http://www.TIMCS.com

The Journal of Mathematics and Computer Science Vol .2 No.2 (2011) 394-401

\title{
Fuzzy approach to Likert Spectrum in Classified levels in Surveying researches
}

\author{
Ahmad Hedayatpanah \\ Sama technical vocational school, Islamic Azad University, Rasht Branch, Rasht, Iran \\ E_Mail; A_hedayatpanah@yahoo.com
}

Received: August 2010, Revised: November 2010

Online Publication: January 2011

\begin{abstract}
Educattion researches in surveying researches of qualifying type are generally exposed to Nominal or Ordinal classified levels. In ordinal level in opinion polling papers, most researchers deal with choices such as very good (very much), good (much), OK (to some extent), poor (little), very poor (very little) and so on. Researchers are forced to analyze the data in order to correspond them to quantity. For this action, the 5-level Likert spectrum (American Sociologist) is used and correspond them to 1, 2, 3, 4, 5 with equal distances of same value, so it used non-parameter tests because of non-parameter data. Since the respondents' view was considered as a kind of show-off, it's obvious that, first, that is a difference between beliefs and views which lead to difficulty in analysis. Second, these is a kind of ambiguity and overlapping in the vicinity of the border of choices that can lead to error in deviation, variance, and test use. This problem also increases the analysis problem and reduces the precision. In this paper, the purpose of writer is to have a fuzzy approach to Likert spectrum and use the fuzzy model for research analysis.
\end{abstract}

Keywords: Fuzzy, Likert, Scales, Spectrum, Surveying research, Opinion polling 


\section{Introduction}

Humanities researchers especially education ones in field researches of surveying research are exposed to 4-level scale called Stevens (American Psychologist). These scales are: Nominal, Ordinal, Interval and Ration. In this paper, classified data especially ordinal ones are investigated. For corresponding the characteristics of variables from quality to quantity, in other words changing the opinion polling choices for measurement and analysis, different spectrums are suggested including the American's Thurstone scale or spectrum which is applied for qualitative categories. It includes 11 equal trends. Later another American sociologist called Likert reduced the bulk of spectrum into 5 trends with equal characteristics for convenience (Taleghani, 2002). Most researchers deal with choices such as very good (very much), good (much), OK (to some extent), poor (little), very poor (very little) in ordinal scale in opinion polling. To analyze the data, researchers are forced to correspond them to quantified form. For this action, mostly 5-level Likert (American sociologist) is used and they correspond them to numbers 1, 2, 3, 4, 5 with equal distances of same value, then because of non-parameter data, they are forced to use non-parameter tests. Since the respondents' view is considered as a show-off, It's obvious that: first, there is a difference in respondents' beliefs and ideas which leads to difficulty in analysis. Second, there is a type of ambiguity and overlap in which can lead to error in deviation, variance, and test use. This also increases the analysis difficulties and decreases the precision. In this paper, the purpose of writer is to investigate 5-level Likert spectrum, in which there is a kind of ambiguity and overlap, from fuzzy view and represent a fuzzy model relying on Likert spectrum for analyzing categories or qualitative characteristics.

\section{Important Definitions:}

A. Nominal Scale: This is the most primitive scale. It just determines the levels (Delavar, 2006). In fact, it's a type of coding scale as Nominal code, sex, martial status, birth place.

B. Ordinal Scale: This scale like nominal one considers classifying and naming besides ordering the levels. It considers ordering people or things from the highest to lowest or vice versa based on characteristics like levels $1,2,3, \ldots$ which are expressed in a competition by ordinal numbers (Hedayatpanah2009). In nominal and ordinal levels, which are famous as classified levels, disjointed numbers in the form of natural and integer number are used. Absolute zero is not defined in them and no mathematical operation is allowed. 
C. Interval Scale: This scale precisely determines the available distances between people, things, or events besides classifying, naming, and ordering like students' psychology score, centigrad degree, thermometer Fahrenheit degree, correlation coefficient, angular tangent such as 45 degrees. There is no absolute zero in this scale. Out of mathematical operations, just adding and subtracting are allowed.

D. Ratiol Scale: This is the most precise and complete scale which includes nominal, ordinal, and interval such as height, weight, and issues which are more physical. We have absolute zero just in this scale and all mathematic operations are possible (Hedayatpanah, 2009). The precision of raito scale is more than interval and the precision of interval scale is more than ordinal and the precision of ordinal scale is more than that of nominal one.

E. Opinion Polling: An information form used for measuring the people's views or beliefs is called Opinion Polling (John Best, 1998).

F. Surveying Research: It's like other methods of research and that's like census. The only difference is that in surveying research a sample of society is selected, while in census the whole community is studied. This research is applied when the researcher intends to collect information such as the numbers of people who agrees or disagrees with a specified belief. Questionnaire and face-to-face interviews are the most usual tools for this method (Esmaeeli, and Biabangard, 2004).

G. Fuzzy Collection (Fuzzy Sets): It's defined based on membership function which is the illustration of a comprehensive collection in (zero and one) interval. Each member has a membership degree. Fuzzy collection is formed out of generalizing classic collections. In classic collections theory, the membership in a set is determined as binary sentences based on binary conditions; While, in fuzzy theory the relative degree of membership in a collection is authorized.(wikipedia)

\section{H. Function and Membership Degree:}

Function is a functional membership of the illustration of the whole collection to $\mathrm{U}^{\prime}$ compared with closed interval $[0,1]$, A fuzzy collection with a membership function $₫ A$ is defined in $U$.

The number that function gives to each member determines the membership degree of that member in collection. If the membership degree of an element in a collection equals zero, that member is completely out of collection, and if the membership degree of an element equals 1 , that member is completely in that collection. We can conclude that classic collection of a fuzzy collection means subset 
of a fuzzy collection, but if the membership degree of an element is between 1 and zero, that number shows the gradual membership rate.

Conceptually, each collection with a degree can interferentially be located in another collection. For example, we can consider age as the lingual variable of youth characteristics with regard to the selection of membership function such as Gavsian, the middle-age characteristics can be located in youth characteristics with a low membership degree and -old-age characteristics appears in youth characteristics with less membership degree.

\section{Fuzzy Reflection}

Bart Kosko believes that it's not possible to divide different phenomena into two categories such as True/False or Zero/One. We should not evaluate the logical and mathematical subjects with such deduction, but we should evaluate everything proportionally. All facts should be evaluated relatively and a rating should be considered for them. In fact, everything is true or false relatively. Most things which seem true are partially true. There is always uncertainty about correctness or incorrectness of factual phenomena. In other words, the factual phenomena are not just black or white, but they are to some extent grey. The factual phenomena are always ambiguous and imprecise. Being fuzzy is the same as being grey. The fuzzy case is an official name in sciences that includes poly-value case. In contrast with fuzzy case, is the two-value case in which for each question, there are two answers: True/False, 1/0. Being fuzzy means being poly-value. This doesn't mean that for each question there are 3 choices or more and perhaps there are endlen choices instead of just two final choices. Each Logical Fuzzy phrase can be to some extent true, the amount of correctness vary between 0 and 1 or it can be true from $0 \%$ to 100\% (Bart Kosko, 2007).

J. Fuzzy Principle: The Fuzzy principle expresses that everything is relative.

K. The difference between classic collection and fuzzy collection: the main reason for classifying classic collection and Fuzzy collection, although there are certain similarities, is the absence of compliance of some rules.

1. In Fuzzy collection theory, membership function is applied.

2. The partnership with its complement is not empty (Rejecting law «the law of excluded middle»).

3. The congregation of collection with its complement doesn't equal with a whole collection. Rejecting the absence of contradiction rule.

\section{Historical investigation of Fuzzy reflection.}


At first, new geometry was represented through rejecting the Euclid parallelism. New algebra also appeared through rejecting the replacing rule in multiplication. In a parallel way, new logic which is famous as multiple logic, at first, appeared through rejecting the Aristotle's middle rejection. Based on this rule, the disjunction proposition is a logical union. In other words, Aristotle's logic is whether correct or incorrect and we don't have anything in the middle for its correctness. In Aristotle's logic, each premise includes just one of these two forms, i.e. correct or incorrect. From this perspective, this logic is famous as two-value logic. In 1921, J. Lukasiewics investigated a 3-value logic. Later on E.L Post studied M-value logic. A. Tarski also studied M-value logic in 1930. Then H. Reichenbach generalized it into the endless value logic in 1932. F. Zwicky understood that poly-value logic can be applied in Quantum Theory (Bijan Zadeh, 1995).

In 1937, a Quantum philosopher called Max Black published a paper about irrational collection or what we, nowadays, call it Fuzzy collection.

In 1965, Lotfizadeh published an article called Fuzzy Collection. In this article J. Lukasiewicz's poly-value logic was used for collection and groups of things. The Aristotle's binary logic ended up into a rule. The sky is blue or not. The sky can't be both blue and non-blue. Two-value logic sacrifices preciseness to easiness. The results of binary logic can simplify the mathematic issues and computer processes into yes/no, white/black, true/false. You see some of these two-values or poly-values in the following:

2-value

poly-value

Aristotle

Buddha

A or non-A

$A$ and non-A

Complete

Slight/Trivial

All or none

To some extent

Zero/One

Continuous amount between 0 and 1 (Bart Kosko, 2007)

If we show the possible value in a conjunction for each premise, in a way that it's located on left side at top, there is 256 possible ways to complete the chart. The following chart is a logical one that Tarski and so on supported it.

\begin{tabular}{|r|r|r|r|}
\hline$\Lambda$ & $\mathrm{T}$ & $?$ & $\mathrm{~F}$ \\
\hline $\mathrm{T}$ & $\mathrm{T}$ & $?$ & $\mathrm{~F}$ \\
\hline$?$ & $?$ & $?$ & $\mathrm{~F}$ \\
\hline $\mathrm{F}$ & $\mathrm{F}$ & $\mathrm{F}$ & $\mathrm{F}$ \\
\hline
\end{tabular}

Fuzzy Approach: 
By a research example, we proceed Likert spectrum with a Fuzzy approach. In a research, the following information was taken from mathematics teachers through the following question.

To what extent are you familiar with Fuzzy reflection?

\begin{tabular}{|c|c|c|c|c|c|c|c|c|}
\hline Average & Total & $\begin{array}{c}\text { Very } \\
\text { little } \\
(1)\end{array}$ & $\begin{array}{c}\text { little } \\
(2)\end{array}$ & $\begin{array}{c}\text { OK } \\
\text { (to some } \\
\text { extent) }\end{array}$ & much & $\begin{array}{c}\text { very } \\
\text { much } \\
(5)\end{array}$ & freguency & Sample \\
\hline $2 / 7$ & 135 & 15 & 7 & 15 & 4 & 9 & F & $\begin{array}{r}\text { Mathematics } \\
\text { Teachers }\end{array}$ \\
\hline
\end{tabular}

As we can't infer from the chart, the researchers use Likert spectrum for chart analysis. Because of this reason, they specify choice very good to number 5 , good to 4 , OK to 3 , and poor and very poor to 2,1 , respectively. There are two important and reflective points in opinion polling paper.

A. The distance between choices in Likert spectrum is equal; while, there is ambiguity in view in which some types of respondents' show-off, it's possible not to have equal values between a respondent's choice good and another's choice.

B. There is also ambiguity between the border of choices and this may lead to disorder in analysis and this itself expresses Fuzzy matters in this spectrum. It's worthy to note that researchers are gradually applying the fuzzy approach, a good example is the analysis of a research done by (Chavoshian and Sotoudeh, 2006) in leveling occupation for nominal data.

If we describe the choices in Likert levels as too little, little, $\mathrm{OK}$, much, too much in the above-mentioned example, the division will be in the following form:

very much much some to text Little Very little
$\left(-{ }^{-}-(--)\right.$

The calculated average equals 2.7. It means:

$$
\bar{x}=\frac{(r \Delta+19+r \Delta+1 \%+1 \Delta)}{\Delta \cdot}=r \cdot r
$$


As you can see, this division is invalid around the borders. But if this division is done by Fuzzy collections, then we won't have border problem, and an idea can be both in very low level and in low level but with different membership like the following figure (Machaichi, 2000):

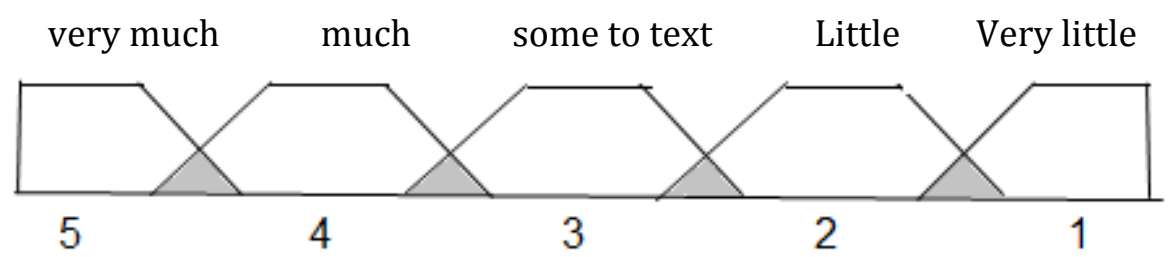

For representing the model, the amount of membership should be clear. This amount is, in fact, for overlapping. If we have suitable theoretical foundation of overlapping kind and amount, by changing this number can reach any favorite overlap. We can calculate the amount of membership by the use of Gous distribution formulae.

$$
f(x)=\frac{1}{\sigma \sqrt{2 \pi}} e^{\frac{1}{2}(x-\mu)^{2} / \sigma^{2}}
$$

In other words, for representing this model, it's necessary to have $\mu, \sigma$ (Chavoshian and Sotoudeh, 2007).

$\bar{x}=\frac{(15 * / 8)+(15 * 1 / 2+(7 * 1 / 8)+(7 * / 2 / 2)+(15 * 2 / 8)+(15 * 3 / 2)+(4 * 3 / 8)+(4 * 4 / 2)+(9 * 5)}{87}=2.58$

Terms like (little, much, too little, too much, to some extent,...) are called linguijtic terms, i.e. we can't specify certain amounts for them with mathematical amounts. It's here that Fuzzy logic is put into use (Askari, Hamed). Based on Fuzzy approach, the average for the above-mentioned example is 2.58 which is calculated with the premise of the overlapping number 0.2. It means there is overlap for each number.

If the selected overlapping number $0 / 1$, the obtained average is also number $2 / 58$. For overlapping numbers between 1 to 5 , the writer did the calculation and the same results were obtained. For $0 / 01$, the result of average didn't change.

\begin{tabular}{|r|r|r|r|r|r|r|}
\hline $\begin{array}{r}\text { Overlap } \\
\text { number }\end{array}$ &.$/ 01$ &.$/ 1$ &.$/ 2$ &.$/ 3$ &.$/ 4$ &.$/ 5$ \\
\hline average & $2 / 58$ & $2 / 58$ & $2 / 58$ & $2 / 58$ & $2 / 58$ & $2 / 58$ \\
\hline
\end{tabular}

Conclusion: For analyzing the data, there are at least two possible methods in front of the researcher. First, it's possible to use non-fuzzy method and it includes ambiguity. The second method is to use Fuzzy variable which is based on Fuzzy logic, it's results are to 
some extent valid and it's ambiguity is less. With regard to overlap in choices, the obtained average in this method is relative, but in Likert Spectrum obtained average is certain .

\section{Reference:}

1. Askari, Hamed. (2008) Wikipedia.

2. Bart Kosko,( 2007). Fuzzy Thinking, Translated by, Ali Ghafari adel .Maghsoodpoor,pour momtaz and Qosimi ,K.N.T University of Technology, Tehran.

3. Bijan Zadeh, (1995) Philosophy of Matematics, Payam Noor University.

4. Chavoshian and Sotoudeh, (2006) ,Fuzzy set applied for ordinary work esteem Rasht city (article).

5. Delavar,( 2006)., Research basic Theory, Roshd Tehran.

6. Esmaeeli, and Biabangard(2004): Statistics and Research methodh,sanjesh Tehran.

7. Hedayatpanah,( 2009), How Research ? daryaye danesh ,Rasht.

8. John Best,(1998).). Research and Education, Translated by. Pasha Sharifi and taleghani .

9. Machaichi, M,(2000):Fuzzy sets, Bahonar University of Kerman.

10. Semnani,D,(2009): Fuzzy logic and fuzzy neural systems.

11. Taleghani, mahmood,(2002), Basic Research Payam Noor University. 